\title{
QUASI-UNMIXEDNESS AND INTEGRAL CLOSURE OF REES RINGS
}

\author{
PETER G. SAWTELLE ${ }^{1}$
}

\begin{abstract}
For certain Rees rings $\Re$ of a local domain $R$, the quasiunmixedness of $R$ is characterized in terms of a certain transform of $R$ being contained in the integral closure of $\Re$.
\end{abstract}

1. Introduction. In this paper, a ring shall be a commutative ring with identity. The terminology is basically that of [2] and [12].

Relations between quasi-unmixedness and integral extensions are well known (e.g., [1], [5] and [7]). Also, the study of properties of a ring $R$ via transition to a Rees ring $\Re=\Re(R, A)$ of $R$ (conditions on the ideal $A$ depending on the particular discussion) has often been useful. In particular, characterizations of the quasi-unmixedness of $R$ are given in [10] in terms of localizations of $\Re$ containing $R$ as a quasi-subspace. The $\Re$-algebra $\mathscr{T}=\mathscr{T}(u \Re)$ (Definition 1) is used in [8] to characterize unmixed local domains. Here, equivalences to the quasi-unmixedness of $R$ are given in terms of $\mathcal{T}$ being contained in the integral closure of $\Re$ (Theorem 2).

2. Preliminary concepts. Let $B=\left(b_{1}, \ldots, b_{k}\right) R$ be an ideal in a Noetherian ring $R$. Let $t$ be an indeterminant, and let $u=1 / t$. The Rees ring $\Re=\mathcal{R}(R, B)$ of $R$ with respect to $B$ is the ring $\Re=R\left[u, t b_{1}, \ldots, t b_{k}\right]$. $R$ is a graded Noetherian subring of $R[u, t]$. If $(R, M)$ is a local ring, then $\Re=(M, u$, $\left.t b_{1}, \ldots, t b_{k}\right)$ is the unique maximal homogeneous ideal of $\Re$. Similar to [12, Theorem 11, p. 157], $\Re^{\prime}$ is a graded subring of $K[u, t]$, where $K$ is the total quotient ring of $R$. (Throughout, $S^{\prime}$ will denote the integral closure of ring $S$.)

For an ideal $B$ in a ring $R$, the integral closure of $B$ in $R$, denoted $B_{a}$, is the set of all elements in $R$ satisfying an equation of the form $x^{n}+b_{1} x^{n-1}+\cdots$ $+b_{n}=0$, where $b_{i} \in B^{i}, i=1, \ldots, n$. It is known [4, p. 523] that $B_{a}$ is an ideal in $R$. In particular, if $B=b R$ is a regular principal ideal, then $(b R)_{a}=\left\{r \in R ; r / b \in R^{\prime}\right\}=b R^{\prime} \cap R[6$, Lemma 1].

DEFINITION 1. Let $b$ be a regular nonunit in a ring $R$. Define $\mathscr{T}(b R)$ $=\left\{c_{k} / b^{k} ; c_{k} \in\left(b^{k} R\right)^{(1)}\right.$, for all large $\left.k\right\}$, where $\left(b^{k} R\right)^{(1)}$ is the set of elements of $R$ that are in each height one primary component of $b^{k} R$.

REMARK. The following are shown in [11].

(1) $\mathscr{T}(b R)$ is contained in $R^{\prime}$ if and only if each height one prime divisor of

Received by the editors July 16, 1973.

AMS (MOS) subject classifications (1970). Primary 13H10, 13C15; Secondary $13 \mathrm{~J} 10$.

Key words and phrases. Local ring, quasi-unmixed, integral closure, Rees ring.

1 This paper contains part of the author's doctoral dissertation written at the University of California at Riverside under the direction of Professor Louis J. Ratliff, Jr. 
$b R^{\prime}$ contracts to a height one prime (divisor of $b R$ ) in $R$.

(2) $b^{n} \mathcal{T}(b R)$ is a finite intersection of height one primary ideals. Also $b^{n} \mathscr{T}(b R) \cap R=\left(b^{n} R\right)^{(1)}$.

(3) Define $R^{(1)}=\bigcap\left\{R_{(P)} ; P\right.$ is a height one prime divisor of a principal ideal generated by a nonzero divisor in $R$, where $(P)$ denotes the set of regular elements in $R-P$. Then $\sigma(b R)=R[1 / b] \cap R^{(1)}$.

3. Characterizations of quasi-unmixed local domains. Several preliminary results on completions are given to show that the condition $\sigma \subseteq \Re^{\prime}$ is equivalent to a similar condition for the completion $R^{*}$ of $R$ (Corollary 1 ). This is used to give equivalences to the quasi-unmixedness of a local domain (Theorem 2).

Lemma 1. Let $B$ be an $M$-primary ideal of a local ring $(R, M)$. Let $\Re=\Re(R, B)$. Let $p$ be a prime ideal of $\Re$ with $u \Re \subseteq p$. Then $(M, u) \Re \subseteq p$, and so all prime ideals containing $u \Re$ lie over $M$.

Proof. Since $u$ is in $p, B=u \Re \cap R \subseteq p \cap R$. But $B$ is $M$-primary, so $M \subseteq p \cap R$, i.e., $M=p \cap R$. Q.E.D.

LEMMA 2. Let $\Re$ be as in Lemma 1 and $\mathcal{S}=\Re\left(R^{*}, B R^{*}\right)$. Let $\Re\left(\right.$ resp., $\left.\mathfrak{R}^{\prime}\right)$ be the maximal homogeneous ideal of $\Re($ resp., $\delta)$, and let $\Re^{*}$ (resp., $\mathcal{S}^{*}$ ) be the completion of $\Re($ resp., $\delta)$ with respect to the $\mathfrak{R}$ (resp., $\left.\mathfrak{R}^{\prime}\right)$-adic topology. Then $\Re^{*}=\mathcal{S}^{*}$ is the completion $\left(\Re_{\Re}\right)^{*}=\left(\delta_{\Re^{\prime}}\right)^{*}$ of $\Re_{\Re}$ and $\delta_{\Re^{\prime}}$.

Proof. $\Re_{\Re}$ is a dense subspace of $\delta_{\mathscr{R}^{\prime}}\left[8\right.$, Lemma 3.2] and $\Re^{*}$ (resp., $\varsigma^{*}$ ) is the natural completion of $\Re_{\Re}$ (resp., $\delta_{\pi^{\prime}}$ ) [3, Theorem 32, p. 434]. Q.E.D.

Lemma 3. Let $R, R^{*}, B, \Re$ and $S$ be as in Lemma 2. Also, assume that $B$ is generated by a system of parameters. Let $\mathcal{T}=\mathcal{T}(u \Re)$ and $\mathcal{T}^{*}=\mathcal{T}(u \delta)$. Then $\left.N=(M, u) \Re_{((M, u))} \cap \mathcal{T}_{(\text {resp., }} N^{*}=\left(M^{*}, u\right) \Re_{\left(\left(M^{*}, u\right)\right)} \cap \mathcal{T}^{*}\right)$ is the only prime divisor of $u \mathcal{T}$ (resp., $\left.u \mathcal{T}^{*}\right)$.

Proof. By [8, Remark 3.10(ii)], $(M, u) \mathcal{R}$ is the only height one prime divisor of $u \Re$. By the one-to-one correspondence (and denseness) in [8, Lemma 3.2], $\left(M^{*}, u\right) \delta=(M, u) \delta^{*}$ is the only height one prime divisor of $u \delta$, and by the one-to-one correspondence in [11, Lemma 2(9)], $N$ (resp., $N^{*}$ ) is the only height one prime divisor of $u \mathscr{T}$ (resp., $u^{*}$ ). By Remark (2), this ideal has no imbedded prime divisors. Q.E.D.

THEOREM 1. With the notation of Lemma 2, let $p \subseteq P$ be an inclusion of prime ideals in $R$ with $u \in p$. Then the following statements hold:

(1) $R / p$ is a locally unmixed, pseudo-geometric domain $[2, p .131]$.

(2) $p \Re_{P}^{*}$ is a semiprime, unmixed ideal in the completion $\Re_{P}^{*}$ of $\Re_{p}$.

(3) In the completion $\Re_{P}^{*}$ of $\Re_{P}, p \Re_{P}^{*}$ has pure height equal to height $p$ and has pure depth equal to depth $p \Re_{P}$.

(4) $p \Re^{*}=p \delta^{*}$ has pure height equal to height $p$, where $p$ is contained in the maximal homogeneous ideal of $R$.

Proof. Since $p \cap R=M($ Lemma 1$), \Re / p=(R / M)\left[u^{\sharp},(t B)^{\sharp}\right]$, where $X^{\sharp}$ denotes $X$ modulo $p$. Thus $\Re / p$ is finitely generated as a ring over the field 
$R / M$, and so is locally unmixed [2, (34.9)], and pseudo-geometric [2, (36.5)]. This shows (1). By localizing to $\Re_{P},(2)$ follows from [2, (36.4)] and (1).

For (3), since $p \Re_{P}^{*}$ is an unmixed ideal (by (2)), it has pure depth equal to depth $p \Re_{P}^{*}=\operatorname{depth} p \Re_{P}$. Since $p \Re_{P}^{*}$ is semiprime, that it has pure height equal to height $p$ follows from [2, (22.9)]. (4) is a special case of (3) since $\Re_{\Re}^{*}=\Re^{*}=\delta^{*}$ by Lemma 2 . Q.E.D.

CoROllaRy 1. Let the notation be as in Lemma 2. Then $\mathscr{T}(u \Re) \subseteq R^{\prime}$ if and only if $\mathscr{T}(u \delta) \subseteq \delta^{\prime}$.

PRoOF. Since $\left(u^{n} \Re\right)_{a}=u^{n} \Re^{\prime} \cap \Re$ and $\Re^{\prime}$ and $\Re$ are graded subrings of $K[u, t]$, it follows that $\left(u^{n} \Re\right)_{a}$ is a homogeneous ideal in $\Re$. Therefore, every prime divisor of $\left(u^{n} \Re\right)_{a}$, for $n \geqslant 1$, and every prime divisor of the homogeneous ideal $u \Re$ is contained in the maximal homogeneous ideal $\Re$ of $\Re$. By [11, Lemma 4(2)], $\mathcal{T}\left(u \Re_{\Re}\right) \subseteq \Re_{\mathscr{N}}^{\prime}$ if and only if $\mathscr{T}(u \Re) \subseteq \Re^{\prime}$. Now, let $P$ be a height one prime divisor of $u \Re_{\mathscr{R}}$, and $p=P \cap \Re$. Then $P \Re_{\mathfrak{R}}^{*}=P \Re^{*}$ has pure height one (Theorem 1(4)). Therefore, by [11, Corollary 2], $\mathcal{T}\left(u \Re_{\Re}\right)$ $\subseteq \Re_{\Re}^{\prime}$ if and only if $\mathcal{T}\left(u \Re_{\Re}^{*}\right) \subseteq \Re_{\mathscr{N}}^{*}$. But $\Re_{\mathscr{N}}^{*}=\left(\delta_{\Re^{\prime}}\right)^{*}$ so the last inclusion is equivalent to $\mathscr{T}\left(u \delta_{\mathscr{N}^{\prime}}\right)^{*} \subseteq\left(\delta_{\mathscr{N}^{\prime}}\right)^{* \prime}$. As above, this is equivalent to $\mathscr{T}\left(u \delta_{\mathscr{N}^{\prime}}\right)$

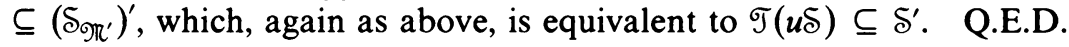

LEMMA 4. Let $b$ be a regular nonunit in a Noetherian ring $R$ and $q$ a minimal prime divisor of zero in $R^{\prime}$. Then there exists a height one prime divisor $P$ of $b R^{\prime}$ that contains $q$.

Proof. In $R^{\prime}$, let $Z=\operatorname{rad}(0)=\bigcap_{i=1}^{n} q_{i}\left(q_{1}=q\right)$. Since $Z \subseteq b R^{\prime}[9$, Lemma 2.4], we may pass to $R^{\prime} / Z=\bar{R}$. $\bar{R}$ is the direct sum of Krull domains $\oplus_{i=1}^{n} R^{\prime} / q_{i}=\oplus_{i=1}^{n} \bar{R} e_{i}$, where the $e_{i}$ are the associated orthogonal idempotents. A height one prime divisor $p_{1}$ of $b e_{1}$ in $\bar{R} e_{1}$ gives rise to the desired $P$. Q.E.D.

THEOREM 2 (cf. [8, Theorem 5.17]). Let $(R, M)$ be a local domain of altitude $n \geqslant 1$. Then the following statements are equivalent:

(1) $R$ is quasi-unmixed.

(2) For every finitely generated domain $A$ over $R$, and for each multiplicatively closed subset $S$ of $A,\left(A_{S}\right)^{(1)} \subseteq A_{S^{\prime}}$.

(3) For every ideal $B$ in $R, \mathscr{T}(u \Re) \subseteq \Re^{\prime}$, where $\Re=\Re(R, B)$.

(4) There exists an M-primary ideal $B$ in $R$ that is generated by a system of parameters such that $\mathcal{T}(u \mathcal{R}) \subseteq \Re^{\prime}$, where $\Re=\Re(R, B)$.

Proof. $(1 \Rightarrow 2)$. By [11, Lemma $1(3)$ and (5)], it is sufficient to show $A^{(1)} \subseteq A^{\prime}$. By [5, Corollary 2.5], $A$ is locally quasi-unmixed. Then, by [7, Theorem 3.8], each height one prime ideal in $A^{\prime}$ contracts to a height one prime in $A$. Thus, by [8, Corollary 5.7], $A^{(1)} \subseteq A^{\prime}$.

$(2 \Rightarrow 3)$. Since $\Re$ is a finite extension of $R, \Re^{(1)} \subseteq \Re^{\prime}$, by hypothesis. And, $\mathcal{T}(u \Re) \subseteq \Re^{(1)}$.

$(3 \Rightarrow 4)$ is obvious.

$(4 \Rightarrow 1)$. Let $B$ be an $M$-primary ideal of $R$ generated by a system of parameters. Let $\mathcal{T} \subseteq \Re^{\prime}$, where $\Re=\Re(R, B)$ and $\mathcal{T}=\mathscr{T}(u \Re)$. By Corollary 1 , $\sigma^{*} \subseteq \delta^{\prime}$, where $\delta=\Re\left(R^{*}, B R^{*}\right)$ and $\mathscr{T}^{*}=\mathscr{T}(u \delta)\left(R^{*}\right.$ is the completion of 
$R$ ). Let $q$ be a minimal prime divisor of zero in $\delta$. Let $q^{\prime}$ be the minimal prime divisor of zero in $\delta^{\prime}$ that lies over $q$ ( $\delta$ and $\delta^{\prime}$ have the same total quotient ring). By Lemma 4, there exists a height one prime divisor $p^{\prime}$ of $u \delta^{\prime}$ that contains $q^{\prime}$. By Remark $1, p^{\prime} \cap \mathcal{S}=p$ is a height one prime divisor of $u \delta$. Hence, $q \subseteq p=\left(M^{*}, u\right) \mathcal{S}$ (Lemma 3$)$. Since $q$ was an arbitrary minimal prime divisor of zero in $\delta, R$ is quasi-unmixed [10, Corollary 9]. Q.E.D.

By combining Theorem 2 and the Remark, further characterizations of the quasi-unmixedness of $R$ can be obtained.

\section{BIBLIOGRAPHY}

1. M. Nagata, On the chain problem of prime ideals, Nagoya Math. J. 10 (1956), 51-64. MR 18, 8.

2. Local rings, Interscience Tracts in Pure and Appl. Math., no. 13, Interscience, New York, 1962. MR 27 \#5790.

3. D. G. Northcott, Lessons on rings, modules, and multiplicities, Cambridge Univ. Press, London, 1968. MR 38 \# 144.

4. J. W. Petro, Some results on the asymptotic completion of an ideal, Proc. Amer. Math. Soc. 15 (1964), 519-524. MR 29 \# 118.

5. L. J. Ratliff, Jr., On quasi-unmixed semi-local rings and the altitude formula, Amer. J. Math. 87(1965), 278-284. MR 31 \#3448.

6. - Note on analytically unramified semi-local rings, Proc. Amer. Math. Soc. 17 (1966), 274-279. MR 32 \#149.

7. - On quasi-unmixed local domains, the altitude formula, and the chain condition for prime ideals. I, Amer. J. Math. 91 (1969), 508-528. MR 40 \# 136.

8. - On quasi-unmixed local domains, the altitude formula, and the chain condition for prime ideals. II, Amer. J. Math. 92 (1970), 99-144. MR 42 \#249.

9. - On prime divisors of the integral closure of a principal ideal, J. Reine Angew. Math. 255 (1972), 210-220. MR 47 \#200.

10. Peter G. Sawtelle, Quasi-unmixed local rings and quasi-subspaces, Proc. Amer. Math. Soc. 38 (1973), 59-64. MR 48 \#6097.

11. Characterizations of unmixed and quasi-unmixed local domains, Ph.D. Dissertation, University of California at Riverside, Riverside, Calif., 1971.

12. O. Zariski and P. Samuel, Commutative algebra. Vol. II, University Series in Higher Math., Van Nostrand, Princeton, N.J., 1960. MR 22 \#11006.

Department of Mathematics, University of Missouri, Rolla, Missouri 65401 